\title{
6. Ueber isomere Diphenylcrotonlactone;
} von Johannes Thicle.

In der vorhergebenden Abhandlung wurde gezeigt, dass der Phenacylhydrozimmtsäure zwei Lactone zugehören, ein $\beta \gamma$ ungesăttigtes labiles und ein $\alpha \beta$-ungesättigtes stabiles, welches aus dem $\beta \gamma$-ungesättigten durch Kochen mit Essigsäureanhydrid entsteht. Ganz ähnliche Verhältnisse zeigen sich bei der Desylessigsăure. Beim Lactonisiren geht diese zuerst in ein bisher unbekanntes Diphenylcrotonlacton über, dem die $\beta \gamma$-ungesättigte Formel zuzuschreiben ist, weil es das erste Umwandelungsproduct der Ketonsäure ist,

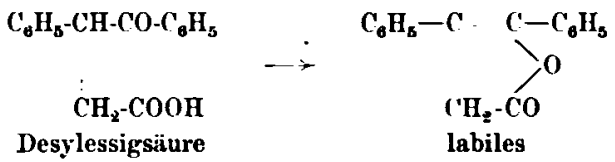

Diphenylcrotonlacton.

Das labile Lacton lässt sich weiterhin in das schon bekannte stabile Lacton der Desylessigsäure uberführen ${ }^{1}$ ). Diesem Lacton ist natürlich bisher die $\beta \gamma$-ungesăttigte Formel zugeschrieben worden, doch ist nicht daran zu zweifeln, dass es analog dem stabilen Lacton der Phenacylhydrozimmtsäure $\alpha \beta$-ungesăttigt ist, nach der Formel:

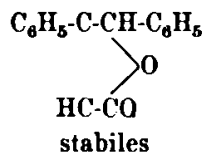

Diphenylcrotonlactou.

Erlenmeyer und $L \mathrm{ux}^{2}$ ) haben dieses Lacton vor einiger Zeit aus dem Diphenyloxybutyrolacton durch Wasserabspaltung erhalten, und unter der damals selbstverständlichen Voraussetzung, dass es $\beta \gamma$-ungesättigt sei, formulirt Erlenmeyer den Lebergang folgendermassen:

1) Klingemann, diese Annalen 269, 131.

q) Ber. d. deutsch. chem. Ges. $31,2224$. 
Thiele, Ueber isomere Diphenylcrotonlactone.

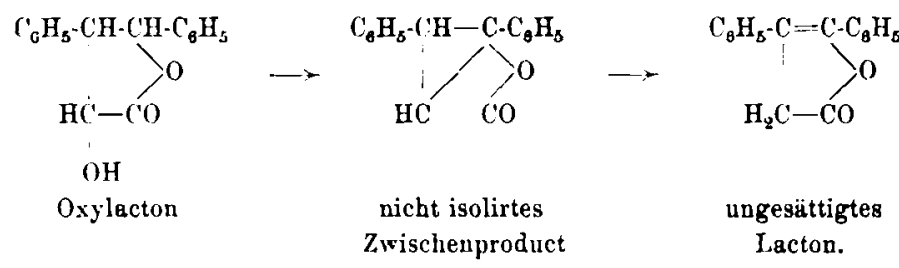

Mit dem Nachweise eines zweiten labilen Diphenylcrotonlactons fält diese Voraussetzung und dann liegt in den Beobachtungen von Erlenmeyer ein starker Beweis dafur, dass das stabile Lacton $\alpha \beta \beta$-ungesăttigt ist,
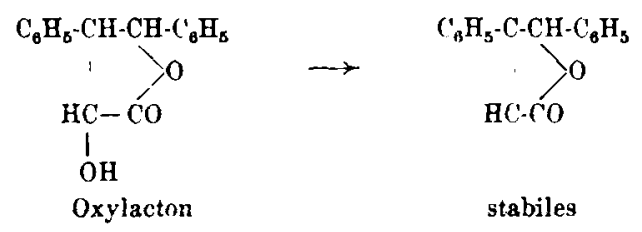

ungesittigtes Lacton.

Für die Lactone der Phenacylhydrozimmtsăure haben die isomeren Diphenylcrotonlactone deswegen Bedeutung, weil für das stabile Lacton der ersteren Säure neben der sehr wahrscheinlichen Formel

I.

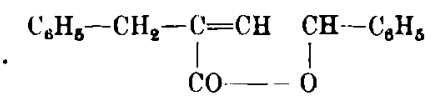

noch die Formel

$$
\text { II. } \begin{gathered}
\mathrm{C}_{8} \mathrm{H}_{8}-\mathrm{CH}=\mathrm{C}-\mathrm{CH}_{2}-\mathrm{CH}^{\prime} \cdot \mathrm{C}_{8} \mathrm{H}_{5} \\
\mathrm{CO}-0
\end{gathered}
$$

in Betracht kam. Dieselbe war dadurch ausgeschlossen worden, dass ein Lacton dieser Formel darstellbar war, welches nicht in Ketonsäure überging.

Eine analoge Formulirung ist für das stabile Diphenylcrotonlacton von vornherein unmöglich. Andererseits ist dasselbe aber das Analogon des stabilen Phenacylhydrozimmtsäurelactons; fur dieses wäre danach also die Formel II noch mehr auszuschliessen, als nach den Ausführungen der vorbergehenden Mittheilung. 
Labiles Diphenylcrotonlacton,<smiles>CC(=O)OC(C)(c1ccccc1)c1ccccc1</smiles>

Fein gepulverte Desylessigsăure wird mit Essigsäureanhydrid ubergossen, dem einige Tropfen Schwefelsäure zugesetzt sind. Die Säure löst sich rasch auf, man giesst dann in Wasser und krystallisirt das bald erstarrende Lacton aus Benzol, oder - bei kleineren Mengen - aus Benzol-Petroläther unter vorsichtigem Erwärmen um. Sehr feine, weisse Nádelchen, die sich zu harten Krusten oder Warzen vereinigen. Schmelzp. 99, $5^{\circ}$ bis $100,5^{\circ}$ unter geringem vorhergehenden Erweichen. Ist das Lacton noch nicht ganz rein, so färbt es sich beim Schmelzen gelb. Leicht löslich in organischen Lösungsmitteln, merklich leichter als das von Klingemann erbaltene stabile Lacton ${ }^{3}$ ).

$$
\begin{aligned}
& 0,1987 \mathrm{~g} \text { gaben } 0,5923 \mathrm{CO}_{2} \text { und } 0,0963 \mathrm{H}_{2} \mathrm{O} \text {. } \\
& 0,2598 \mathrm{~g} \quad \text { in } 21,421 \mathrm{~g} \text { Eisessig } 0,218^{\circ} \text { Depression. } \\
& 0,4854 \mathrm{~g} " \text { in } 21,421 \mathrm{~g} \text { Eisessig } 0,413^{\circ} \text { Depression. } \\
& \text { Berechnet für } \\
& \mathrm{C}_{16} \mathrm{H}_{12} \mathrm{O}_{2} \\
& \text { C } \quad 81,35 \\
& \mathrm{H} \quad 5,08 \\
& \text { Mol.- Gew. } 236 \quad 217214 \\
& 81,22
\end{aligned}
$$

Löst man das Lacton in Alkohol, so fărbt sich dio Lösung auf Zusatz von alkoholischem Kali braun, nach kurzer Zeit bleibt die Lösung mit Wasser klar und Säuren făllen dann Desylessigsäure; Schmelzp. $162^{\circ}$ nach dem Umkrystallisiren.

Stabiles Diphenylcrotonlacton,<smiles>O=C1OC(c2ccccc2)C2CCCCC12</smiles>

Kocht man das labile Lacton $1 \frac{1}{9}$ Stunden mit Essigsăureanhydrid, so färbt sich die Lösung gelb, durch Eingiessen in

3) Diese Annalen 260, 131. 
Thiele u. Schleussner, Phenylcinnamenylacrylsäure etc. 197

Wasser fällt das stabile Lacton aus. Schmelzp. $152^{\circ}$ (nach dem Umkrystallisiren aus Benzol). Weisse, weiche Nädelchen. Kali wandelt den Körper unter denselben Erscheinungen wie bei dem labilen Lacton in Desylessigsäure um. Die Verbindung ist also identisch mit dem von Klingemann erhaltenen Diphenylcrotonlacton.

I. $0,1390 \mathrm{~g}$ gaben $0,4145 \mathrm{CO}_{4}$ und $0,0680 \mathrm{H}_{8} \mathrm{O}$. $0,2438 \mathrm{~g} \quad, \quad$ in $21,271 \mathrm{~g}$ Eisessig $0,193^{\circ}$ Depression.

II. $0,4620 \mathrm{~g} "$ in $21,271 \mathrm{~g}$ Fisessig $0,368^{n}$ Depression.

\begin{tabular}{lcrc} 
& Berechnet für & \multicolumn{2}{c}{ Gefunden } \\
\cline { 3 - 5 } & $\mathrm{C}_{\mathbf{1 0}} \mathrm{H}_{\mathbf{1 2}} \mathrm{O}_{\mathbf{2}}$ & I. & II. \\
$\mathrm{C}$ & $81,3 \tilde{5}$ & 81,33 & - \\
$\mathrm{H}$ & 5,08 & 5,43 & -- \\
Mol.-Gew. & 236 & 230 & 232
\end{tabular}

Die Beziehungen der beiden Lactone sollen noch naher untersucht werden.

7. Zur Darstellung der Phenylcinnamenylacrylsăure und des Diphenylbutadiens; von Johannes Thiele und Karl Schleussner.

Die Phenylcinnamenylacrylsäure,

$$
\begin{gathered}
\mathrm{C}_{8} \mathrm{H}_{8}-\mathrm{C}=\mathrm{CH}-\mathrm{CH}=\mathrm{CH}-\mathrm{C}_{8} \mathrm{H}_{5} \\
\mathrm{COOH}
\end{gathered},
$$

ist schon von Rebuffat ${ }^{1}$ ) durch Condensation von Zimmtaldehyd mit Phenylessigsăure und Reinigung uber das sebr schwer lösliche Baryumsalz erhalten worden, doch lässt sich diese Darstellungsmethode noch sehr verbessern.

\section{Phenylcinnamenylacrylsäure.}

$52 \mathrm{~g}$ phenylessigsaures Natrium, $39 \mathrm{~g}$ Zimmtaldebyd und $80 \mathrm{~g}$ Essigsäureanbydrid werden vier Stunden im Oelbade auf

1) Gazz. chim. ital. 15, 107; 20, 1054. 